\title{
ON VARIATIONS IN THE FLARE ACTIVITY OF V1054 OPH
}

\author{
W. E. KUNKEL
}

Cerro Tololo Inter-American Observatory*, La Serena, Chile

Four years ago I examined the flare incidence data then available for UV Ceti and V1054 Oph (Wolf 630) and concluded that no evidence could be found for periodic or secular variation in the levels of activity (Kunkel, 1971). Variations greater than $0.3 \mathrm{mag}$. (rms) should have been found.

The situation for both stars has changed since then. For V1054 Oph the data volume is about double that available earlier, and second, it is now fairly clear that the coefficient $a$ in the rate equation

$$
R(U)=\exp \left[a\left(U-U_{0}\right)\right] \mathrm{h}^{-1}
$$

does not vary with time, and is the same for all stars, so that it is no longer necessary to solve for both $a$ and $U_{0}$ once for each observing season. Using a mean value of $a$. for all seasons, the precision in estimating $U_{0}$ is somewhat improved.

About 120 ultraviolet flares of V1054 Oph have been reported by MacConnell (1968) and Kunkel (1973). Levels of activity for five epochs have been calculated and plotted in Figure 5 of Kunkel's (1975) review paper. The mean activity is at $U_{0}=14.12 \pm 0.13$. The variance is about double that expected for activity at a constant level, and the figure suggests a weak periodic variation between one and two years.

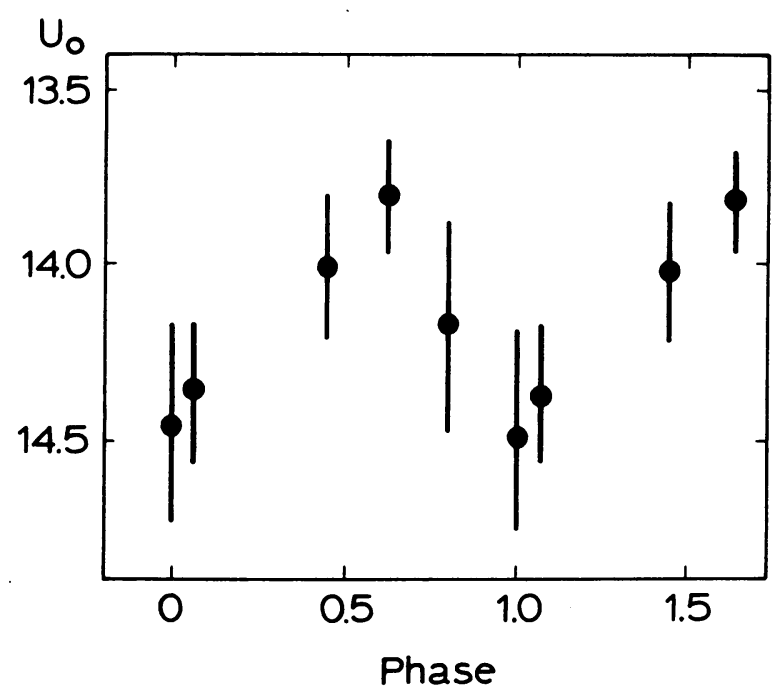

Fig. 1. Flare activity $U_{0}$ of $\mathrm{V} 1054 \mathrm{Oph}$ as a function of phase according to the orbital period of 1.715 yrs.

* Operated by the Association of Universities for Research in Astronomy, Inc., under contract with the National Science Foundation. 
V1054 Oph is a visual binary with a period of 1.715 yr (Baize, 1966), close to the period suggested in the variation of flare activity. The flare incidence data have been replotted in a phase diagram based on the orbital period (Figure 1). The satisfactory agreement implies that the periodic variation of activity, if real, may be coupled to the orbital period of this binary.

Considerable caution must be excercised in assessing this still preliminary result. Observational errors are large, leaving a probability of about $70 \%$ that the result is real, or 30 that it is spurious. Observations of about 30 flares per season are required over a lapse of three years to confirm or reject this result with confidence.

A continuation of this investigation is of great interest, since there are some indications that duplicity may be important in extending the flare active phase of stellar evolution. V1054 Oph has been identified by Eggen (1965) as a member of a moving group with a color-magnitude diagram resembling that of M67. Given the spectral type (dM3.5e) and luminosity $\left(M_{v}=10.7\right)$ of V1054 Oph, this is the oldest flare star with a clear indication of age, and so may be taken to represent a situation in which the mechanism for prolonging the flare active phase is important.

\section{References}

Baize, P.: 1966, J. Obs. 49, 1.

Eggen, O. J.: 1965, Observatory 85, 191.

MacConnell, D. J.: 1968, Astrophys. J. 153, 313.

Kunkel, W. E.: 1971, Bull. Am. Astron. Soc. 3, 13.

Kunkel, W. E.: 1973, Astrophys. J. Suppl. 25, 1.

Kunkel, W. E.: 1975, this volume, p. 15. 